\title{
MEDICAMENTOS SUJEITOS A CONTROLE ESPECIAL MAIS UTILIZADOS EM CENTROS DE ATENÇÃO PSICOSSOCIAL EM UMA CIDADE DO PARANÁ
}

\section{DRUGS OF SPECIAL CONTROL MOST USED IN PSYCHOSOCIAL CARE CENTER IN A CITY OF PARANÁ}

\section{Beatriz BÖGER ${ }^{\star 1}$, Chayanne FEDERHEN ${ }^{2}$, Mayara BRAND², Renata SZPAK'; Beatriz PATRIOTA $^{3}$, Linda MORISHITA ${ }^{3}$, Eliane Carneiro GOMES ${ }^{1}$}

\author{
1 - Programa de Pós Graduação em Ciências Farmacêuticas- UFPR \\ 2 - Departamento de Farmácia- UFPR \\ 3 - Secretária Municipal de Saúde de Curitiba- PR \\ *Autor para correspondência: beatrizboger@gmail.com.
}

\begin{abstract}
RESUMO:
O objetivo do trabalho foi identificar os medicamentos sujeitos a controle especial mais utilizados em Centros de Atenção Psicossocial (CAPS) no município de Curitiba, ParanáBR. Estudo retrospectivo, por meio de pesquisa documental em Livros de Registro Específico de medicamentos controlados dos CAPS, referente aos anos de 2013 a 2016. Constatou-se a saída de 10.897 medicamentos, sendo as dispensações para uso emergencial. Foi considerada a denominação comum brasileira (DCB), a forma farmacêutica e as concentrações que eram dispensadas das seguintes classes terapêuticas: antidepressivos, ansiolíticos, antiepiléticos, antipsicóticos, anticolinérgicos e hipnóticos/sedativos. Os mais utilizados foram os antipsicóticos, em específico o Cloridrato de Clorpromazina em comprimido de 100mg (23,41\%) e 25mg (18,54\%), seguido do ansiolítico Diazepam em comprimido de $5 \mathrm{mg}(11,86 \%)$. O padrão de consumo de psicotrópicos aqui identificados não diferem muito dos estudos semelhantes em outras regiões do Brasil, além de evidenciar o consumo em caráter emergencial de algumas classes terapêuticas.
\end{abstract}

Palavras-chaves: medicamentos de controle especial; psicotrópicos; saúde mental; serviços de saúde; saúde pública.

\section{ABSTRACT}

This study aimed to analyze the most used psychotropic drugs in instrument of Psychosocial Network, the Psychosocial Care Center (CAPS) in Curitiba, Paraná- BR. We performed a transverse quantitative analysis, through the search for information in the Specific Registration Books of controlled drugs in the CAPS in the years 2013 to 2016. Where were dispensed 10.897 drugs for emergency use in mental disorders. Took into account the Brazilian common name (DCB), pharmaceutical form and concentrations that are exempted from the following drug classes: antidepressants, anxiolytics, antiepileptics, antipsychotics, anticholinergics, and hypnotic/sedative. The therapeutic class more dispensed was antipsychotics, in specific Chlorpromazine Hydrochloride pill 100mg $(23,41 \%)$ e $25 \mathrm{mg}(18,54 \%)$, followed by anxiolytic Diazepam $5 \mathrm{mg}$ pill $(11,86 \%)$. The standard identified here psychotropic consumption agree with those from similar studies in other regions of Brazil, also highlights showed consumption on an emergency basis for some therapeutic classes. 
Keywords: drugs of special control; psychotropic drugs; mental health; health services; public health.

\section{INTRODUÇÃO}

De acordo com a Portaria MS/ SVS n $344 / 1998$ as substâncias controladas ou sujeitas a controle especial são, em sua maioria, substâncias com ação no Sistema Nervoso Central, capazes de causar dependência física ou psíquica, motivo pelo qual necessitam de um controle mais rígido do que o controle existente para as substâncias comuns. Também se enquadram na classificação de substâncias controladas, os anabolizantes, os abortivos ou que causam malformação fetal, substâncias que podem originar psicotrópicos, insumos utilizados na fabricação de entorpecentes e psicotrópicos, plantas utilizadas na fabricação de entorpecentes, bem como os entorpecentes, além de substâncias químicas de uso das forças armadas e as substâncias de uso proibido no Brasil. Ainda, pela Portaria MS/SVS n. - 344/98, definem-se as seguintes listas de substâncias: A1 e A2 (entorpecentes); A3, B1 e B2 (psicotrópicas); C1 (outras substâncias sujeitas a controle especial); C2 (retinóicas); C3 (imunossupressoras); C4 (anti-retrovirais); C5 (anabolizantes); D1 e D2 (precursores de entorpecentes e psicotrópicos); E (plantas) e F (proscritas) (BRASIL, 2016).

Cabe aqui definir também o termo "psicofármaco" $(p s i c o=$ alma, fármaco $=$ medicamento) que se refere a substâncias psicoativas, as quais, segundo a Organização Mundial da Saúde (OMS) alteram o humor, a cognição e o comportamento, ou seja, afetam a nossa mente. Entre as substâncias psicoativas existem aquelas que também possuem a propriedade de induzir estado de dependência. As substâncias deste grupo recebem a denominação de "psicotrópicos". Os medicamentos psicotrópicos (psique = mente, topos = mudança, iço= relativo a) são modificadores seletivos do Sistema Nervoso Central e podem ser classificados, segundo a OMS em: ansiolíticos e sedativos; antipsicóticos (neurolépticos); antidepressivos; estimulantes psicomotores; psicomiméticos e potencializadores da cognição (RANG, 2004; WHO, 2001).

No mundo, cerca de 450 milhões de pessoas possuem algum tipo de transtorno mental ou comportamental e há expectativa de que esses números cresçam significativamente nos próximos vinte anos (WHO, 2003). No tratamento de tais distúrbios são empregados os psicofármacos, cujo uso vem aumentando nos últimos tempos. Tal fato pode ser uma consequência do crescimento no número de diagnósticos de transtornos mentais, do surgimento de novos psicofármacos e de novas indicações terapêuticas para 
fármacos antigos (RODRIGUES, 2006).

No Brasil, o cuidado em saúde mental no âmbito do Sistema Único de Saúde (SUS) é realizado por meio da Rede de Atenção Psicossocial (RAPS) composta por pontos de atenção à saúde para pessoas com sofrimento ou transtorno mental e com necessidades decorrentes do uso de crack, álcool e outras drogas. O modelo de atenção da RAPS busca assegurar os direitos humanos, através da garantia da liberdade e autonomia dos indivíduos, da atenção humanizada e da inserção social dos sujeitos (BRASIL, 2011).

Esta Rede visa o cuidado integral por meio da assistência multiprofissional, conforme a lógica interdisciplinar, e por ações intersetoriais dos serviços organizados regionalmente. Ela também é composta por diversos equipamentos correspondentes aos níveis de complexidade do atendimento em saúde. Existem os pontos de atendimento da atenção básica, da atenção psicossocial especializada, da atenção de urgência e emergência, da atenção residencial de caráter transitório, da atenção hospitalar, de estratégias de desinstitucionalização e de reabilitação psicossocial (BRASIL, 2011).

Os Centros de Atenção Psicossocial (CAPS) são os serviços de referência em atenção psicossocial especializada. Eles são compostos por equipes multiprofissionais que trabalham de forma interdisciplinar e são articulados com outros pontos da RAPS. Tais Centros atuam de modo territorializado, aberto e comunitário (BRASIL, 2011).

Os CAPS podem ser organizados em diferentes modalidades de acordo com o perfil do usuário, o contingente populacional a ser coberto e o período de funcionamento. Conforme a Portaria do Ministério da Saúde oㅜ 3.088/2011, eles são organizados nas seguintes modalidades:

- CAPS I: atendimento a indivíduos com transtornos mentais graves e persistentes, assim como para aqueles com necessidades decorrentes do uso de crack, álcool e outras drogas de todas as faixas etárias. Recomendado para municípios que possuam população acima de 20 mil habitantes.

- CAPS II: atendimento ao mesmo público que o CAPS I, difere apenas no fato de ser indicado para municípios que apresentem mais de 70 mil habitantes.

- CAPS III: destinado ao atendimento de indivíduos com transtornos mentais graves e persistentes. Oferece serviços de atenção contínua, atendendo 24 horas, inclusive em feriados e finais de semana. É aconselhado para municípios ou regiões que apresentem população maior que 200 mil habitantes.

- CAPS AD: voltado para adultos ou crianças e adolescentes (conforme normativas do Estatuto da Criança e do Adolescente), que apresentem necessidades decorrentes do 
uso de crack, álcool e outras drogas. Possui recomendação para municípios ou regiões com população maior que 70 mil habitantes.

- CAPS AD III: o público atendido é o mesmo do CAPS AD, difere pelo fato de oferecer cuidados clínicos contínuos, com atendimento 24 horas (inclusive feriados e finais de semana). Possui no máximo doze leitos para observação e monitoramento. Preconizado para municípios ou regiões com população de mais de 200 mil habitantes.

- CAPS Infantil: destinado a crianças e adolescentes com transtornos mentais graves e persistentes, assim como aos que fazem uso de crack, álcool e outras drogas. Aconselhado para municípios ou regiões com população acima de 150 mil habitantes.

O município desempenha um papel fundamental na rede, além de obter autonomia para definir a sua própria lista de medicamentos Relação Municipal de Medicamentos (REMUNE) baseada na RENAME (Relação Nacional de Medicamentos Essenciais), ampliando o acesso aos fármacos na rede pública, facilitando a adesão ao tratamento a todos os usuários do SUS e garantindo medicamentos eficazes, seguros e com qualidade (BRASIL, 2013; CASTRO, 2000).

O medicamento é um recurso terapêutico essencial à população e assegurar uma melhor relação custo/efetividade muito importante para adesão farmacoterapeutica (BRASIL,2013). Existe a necessidade de uma política municipal de medicamentos para assegurar a assistência prestada e um arsenal terapêutico necessário e adequado ao atendimento dos principais problemas de saúde de acordo com o cenário sanitário do município. Ter uma lista padronizada de medicamentos é um mecanismo que auxilia na qualidade da assistência farmacêutica a ser prestada e ter uma visão construtiva de sustentabilidade do sistema de saúde ao nível municipal (CASTRO, 2000).

Neste contexto, a presença de psicofármacos na RENAME, assim como nas relações municipais e estaduais, é de grande valia como parte do tratamento dos transtornos mentais, auxiliando a reduzir ou eliminar a incapacitação e outros agravos.

A OMS lembra a importância desses medicamentos ao afirmar que devem estar presentes nas listas de medicamentos essenciais e ser fornecidos em todos os níveis de cuidados à saúde. Os psicofármacos podem aliviar sintomas, contribuir para a retomada da autonomia dos pacientes, abreviar o curso dos transtornos e prevenir recorrências (WHO, 2001). Contudo, seu uso deve ser feito de maneira racional, pois alguns deles podem ocasionar dependência física ou psíquica. Partindo desse ponto de vista as discussões sobre o uso consciente de medicamentos psicofármacos devem estar presentes na rotina da equipe de saúde responsável pela administração, a partir da prescrição, e por fornecer 
instruções aos usuários de forma esclarecedora e objetiva (LOPES, 2011).

Deste modo, o objetivo deste trabalho foi identificar os medicamentos, sujeitos a controle especial, mais utilizados na farmacoterapia emergencial em Centros de Atenção Psicossocial (CAPS) da cidade de Curitiba/PR no período compreendido entre 2013 a 2016.

\section{METODOLOGIA}

A pesquisa quantitativa foi realizada em dois Centros de Atenção Psicossocial (CAPS) em Curitiba/PR, que são pontos de referência dentro da Rede de Atenção Psicossocial (RAPS). Um desses locais era caracterizado, nos anos de 2013 a 2016, como CAPS III voltado para a área de Álcool e outras Drogas (AD), especificamente para o público infanto-juvenil, mas, em 2014, passou a atender Transtornos Mentais em geral, mantendo a mesma faixa etária dos usuários atendidos. Já o outro CAPS de estudo, enquadrava-se como CAPS II durante o período de 2013 a 2016 e passou para a categoria CAPS III em 2014, sempre com o atendimento voltado aos Transtornos Mentais (TM) em geral para o público adulto.

Tratou-se de um estudo retrospectivo a partir de dados secundários. Foram utilizados os Livros de Registro Específico de Medicamentos Controlados de cada um dos dois CAPS pesquisados, para a coleta de dados referentes ao período de 2013 a 2016 . Os medicamentos citados nos livros e, portanto, pesquisados se enquadravam como "Substâncias Sujeitas a Controle Especial" e como "Psicotrópicos" de acordo com a Portaria nº 344 de maio de 1998. Foram consideradas a denominação comum brasileira (DCB), a forma farmacêutica e as concentrações dos medicamentos dispensados.

A busca de informações sobre a dispensação foi realizada por sujeitos envolvidos em um grupo multiprofissional do Programa de Educação pelo Trabalho para a Saúde que contemplou o campo de atuação profissional no SUS.

A coleta de dados para este trabalho recebeu autorização da Secretaria Municipal de Saúde de Curitiba (SMS), igualmente responsável pela indicação dos CAPS a serem estudados. Isto se deve ao fato de que o mesmo faz parte das atividades realizadas no PET-Saúde, desenvolvido por UFPR/SMS/MS - 2013-2016, voltado para a Rede de Atenção Psicossocial (RAPS), o qual teve projeto de pesquisa atrelado e aprovado pelos Comitês de Ética da Universidade Federal do Paraná (CAAE $n^{\circ}$ 38629314.0.0000.0102) e SMS (Protocolo 16/2015) (UFPR, 2013). 


\section{RESULTADOS E DISCUSSÃO}

No CAPS voltado ao atendimento do público adulto, atualmente CAPS III, os medicamentos mais utilizados durante o período de 2013 a 2016, para um total de registro de saída de 2450 unidades, foram: Haloperidol Decanoato $50 \mathrm{mg} / \mathrm{ml}$ injetável $(26,98 \%$ registro de saída de 661 unidades); Diazepam $5 \mathrm{mg}$ comprimido (18,73\% - registro de saída de 459 unidades); Biperideno Lactato $5 \mathrm{mg} / \mathrm{mL}$ injetável (12,82\% - registro de saída de 314 unidades). Pode ser observada utilização desses medicamentos e sua respectiva classe terapêutica na TABELA 1.

TABELA 1: Medicamentos mais utilizados no CAP III e sua respectiva classe terapêutica.

\begin{tabular}{ccc}
\hline $\begin{array}{c}\text { Medicamentos (forma farmacêutica- } \\
\text { concentração) }\end{array}$ & Classe terapêutica & N(\%) \\
\hline Haloperidol Decanoato (ampola $1 \mathrm{~mL}-50 \mathrm{mg} / \mathrm{ml}$ ) & Antipsicótico & $661(26,98 \%)$ \\
Diazepam 5mg comprimido & Depressor do SNC* & $459(18,73 \%)$ \\
Biperideno Lactato (ampola $1 \mathrm{~mL}-5 \mathrm{mg} / \mathrm{mL}$ ) & Anticolinérgico & $314(12,82 \%)$ \\
\hline
\end{tabular}

${ }^{*}$ SNC: Sistema nervoso central.

O Haloperidol é um antipsicótico convencional da classe das butirofenonas. Os antipsicóticos são utilizados no tratamento das psicoses, sendo elas a esquizofrenia e síndromes relacionadas, que podem apresentar situações agudas (crises psicóticas) e situações crônicas (surtos recorrentes, em certos intervalos de tempo). O Haloperidol tem indicação terapêutica para o tratamento de crises relacionadas ao uso de álcool e drogas e para o controle da esquizofrenia e de outros transtornos psicóticos, sendo mais utilizado no segundo caso (SESA, 2005). Ele é injetável quando na forma decanoato. Nesse caso, normalmente é escolhido para utilização por período prolongado, sendo uma boa opção para casos em que não há adesão ao tratamento por via oral. Também é empregado para controle da esquizofrenia e outros transtornos psicóticos, como indicando no Protocolo Clínico e Diretrizes Terapêuticas da Secretária de Estado da Saúde do Paraná (SESA, 2014; BRASIL, 2010).

Os antipsicóticos convencionais ou típicos foram os precursores de seu grupo farmacológico, contudo possuem alguns efeitos colaterais característicos conhecidos como efeitos extrapiramidais, caracterizados pelas distonias agudas e pela discinesia tardia. As distonias agudas são movimentos involuntários, como torcicolo, espasmos musculares, tremores, rigidez, língua protusa, entre outras, além da síndrome parkinsoniana. É 
característico do início do tratamento e tende a regredir com o tempo, sendo reversível com a interrupção da medicação. A discinesia tardia também se caracteriza por movimentos involuntários, nesse caso de face, língua, tronco e membros. Contudo, esse quadro pode ser severamente incapacitante e não é reversível com a interrupção do tratamento, podendo até mesmo piorar. A discinesia tardia pode manifestar-se após meses ou anos de tratamento. É mais comum entre pacientes acima dos 50 anos e depende da substância usada (RANG, 2004).

Em um trabalho realizado para avaliação da utilização de medicamentos sujeitos a controle especial em CAPS no município de Porciúncula/RJ, observou-se que o Haloperidol (via oral) foi o mais prescrito $(43,75 \%)$, assim como encontrado nesta pesquisa no CAPS adulto de Curitiba-PR, porém, na forma injetável, seguido do Decanoato de Haloperidol intramuscular (21,88 \%) e da Clorpromazina (20,83 \%). Por apresentar ação prolongada, o Decanoato de Haloperidol, melhora a adesão de pacientes com esquizofrenia ao tratamento, pelo fato de manter os níveis plasmáticos e evitar re-hospitalizações (BAROZA, 2012).

O Diazepam, por sua vez, é um depressor do sistema nervoso central, da classe dos benzodiazepínicos de ação ansiolítica, hipnótica, sedativa, amnésica, anticonvulsivante e relaxante muscular. É utilizado em emergências de mal epilético, sendo o benzodiazepínico de primeira escolha nesse caso. Também possui indicação terapêutica em transtorno do pânico. Há indícios de que a prescrição de Diazepam corresponda a 50\% das prescrições de psicofármacos no Brasil, sugerindo uma possível sobreprescrição ou até mesmo um uso abusivo desse fármaco (GILMAN, 2006; RANG, 2004; BAROZA, 2012).

Avaliando-se o uso de medicamentos psicotrópicos no serviço municipal de saúde, de Coronel Fabriciano/MG e Diamantina/MG, foram analisadas as prescrições e constatouse que o Diazepam obteve um percentual 59,7 \% e 37,04\%, respectivamente, dos medicamentos mais dispensados. O que diferem do nosso estudo, onde o Diazepam correspondeu a 18,73\% (FILHO, 2011; FIRMINO, 2012).

O alto consumo e a boa aceitação da classe dos benzodiazepínicos podem se justificar pela imagem positiva dessa classe terapêutica por usuários crônicos, em que se destacam alguns pontos positivos: proporcionam efeito relaxante, calmante, sono restaurador e induzem ao sono rapidamente e são considerados eficazes, seguros, de baixo custo, além de possuírem grande popularidade (FILHO, 2011; ORLANDI, 2011).

Além disso, justifica-se a alta prevalência de uso desses fármacos em municípios do Brasil, por estarem presentes na RENAME dispensados gratuitamente mediante a apresentação de receita especifica (FILHO, 2011). 
Já o Biperideno (lactato) é um anticolinérgico com ação majoritariamente central que evita efeitos adversos periféricos; logo, ele é indicado para contornar os efeitos extrapiramidais dos antipsicóticos, o que justifica seu uso pronunciado junto ao Haloperidol (SESA, 2015; BRASIL, 2010).

No estudo realizado no município de Porciúncula/RJ o Biperideno foi utilizado por 22,9\% dos pacientes em associação com antipsicóticos como o Haloperidol (BAROZA, 2012). Comparado com o estudo atual o Biperideno foi o terceiro mais fornecido $(12,82 \%)$, provavelmente porque as drogas anticolinérgicas como o Biperideno são quase quatro vezes mais prescritas aos pacientes em uso do Haloperidol, quando comparado com outras drogas antipsicóticas como, por exemplo, a Olanzapina, pois controlam os efeitos colaterais do Haloperidol, principalmente os sintomas extrapiramidais (OLIVEIRA, 2000).

O Haloperidol e o Biperideno são muito usados em combinação, presume-se que o intenso uso de Haloperidol e suas associações sejam pelo seu custo mais baixo. Analisando o custo desses medicamentos pode-se verificar que esta associação apresenta um custo cerca de três vezes inferior ao uso de um antipsicótico mais moderno (monoterapia) que possui menos efeitos indesejáveis (OLIVEIRA, 2000; BAROZA, 2012).

Em relação ao CAPS responsável pelo atendimento voltado ao público infantojuvenil, os resultados dos psicofármacos mais usados durante o período de 2013 a 2016, de um total de registro de saída de 8447 unidades (TABELA 2), foram: Cloridrato de Clorpromazina comprimido 100mg (28,22\% - 2384 unidades); Cloridrato de Clorpromazina comprimido 25mg (23,45\% - 1981 unidades); Diazepam 5mg comprimido (9,86\% - 833 unidades) e outros psicofármacos ( $38,47 \%$ - 3249 unidades).

TABELA 2: Medicamentos mais utilizados no CAP infanto-juvenil e sua respectiva classe terapêutica

\section{Medicamentos (forma}

farmacêutica- concentração)

Cloridrato de Clorpromazina comprimido $100 \mathrm{mg}$

Cloridrato de Clorpromazina comprimido $25 \mathrm{mg}$

Diazepam $5 \mathrm{mg}$ comprimido
Classe terapêutica

Antipsicótico

Antipsicótico

Depressor do SNC*
$N(\%)$

$2384(28,22 \%)$

$1981(23,45 \%)$

$833(9,86 \%)$

${ }^{*}$ SNC: Sistema nervoso central. 
O Cloridrato de Clorpromazina, assim como o Haloperidol, é um antipsicótico convencional ou clássico, sendo assim, em geral, apresenta as mesmas características acima citadas. Porém, a Clorpromazina faz parte da classe das fenotiazinas. Outra diferença se deve ao fato de que ela é mais apropriada para fase aguda, enquanto o Haloperidol também é adequado para controle em longo prazo. Tem indicação para quadros psicóticos, agitação psicomotora e fase aguda da mania (SESA, 2015).

O benzodiazepínico Diazepam já foi anteriormente apresentado, mas em se tratando de dependência química é indicado nos casos de contenção química em quadros de intoxicação aguda por substâncias psicoativas e para sintomas da síndrome de abstinência do álcool e de hipnóticos-sedativos. O Diazepam previne e controla o delirium tremens da síndrome de abstinência (SESA, 2015).

Estudo avaliando a dispensa de psicotrópico, no município de Anápolis/GO ano de 2011, a classe de antiepiléticos (Carbamazepina) apontou o maior número de prescrições, totalizando $35,33 \%$, enquanto o Diazepam representou $23 \%$ das prescrições (RIBEIRO, 2011).

Não há um fármaco único capaz de tratar a dependência química que é uma doença crônica caracterizada como síndrome comportamental na qual o uso da droga adquiriu prioridade na vida do sujeito causando-lhe comprometimentos relevantes. Por isso, a farmacoterapia é definida a partir da sintomatologia do paciente e pode combinar diferentes medicamentos. Sendo assim, a abordagem da dependência química em protocolos e diretrizes de saúde mental é delineada de acordo com a substância que levou à dependência. O uso ocasional, o uso abusivo e a dependência química de substâncias psicoativas envolvem múltiplos fatores, como por exemplo: sociais, culturais, biológicos e psicológicos. Logo, o tratamento da dependência química também é complexo, envolvendo saberes multidisciplinares e ações multiprofissionais (GRAEFF, 2012).

Avaliando, em conjunto, os registros de utilização de medicamentos de ambos os CAPS, verificou-se que os medicamentos mais utilizados durante o período de 2013 a 2016 , em relação ao total de registro de saída de 10.897 unidades, foram: Cloridrato de Clorpromazina comprimido 100mg (23,41\% - 2551 unidades); Cloridrato de Clorpromazina comprimido 25mg (18,54\% - 2020 unidades); Diazepam 5mg comprimido (11,86\% - 1292 unidades).

Em outro estudo realizado com 30 CAPS da região sul do Brasil por Kantorski et al (2011), o haloperidol apareceu como o medicamento mais utilizado (10,6\%), assim como no CAPS voltado ao público adulto avaliado nessa pesquisa. Nesse mesmo trabalho, o 
Diazepam também figurou entre os mais utilizados (4ำ lugar - 7,5\%), em consonância com os dois CAPS avaliados nesse trabalho. Um dado curioso é o fato de que a classe dos antidepressivos foi a mais utilizada no estudo para o sul do país, sendo a fluoxetina $\circ 2^{\circ}$ medicamento mais utilizado $(9,8 \%)$, percentual bem próximo ao medicamento mais usado, o Haloperidol (10,6\%). Porém, nesta pesquisa, os antidepressivos não apareceram entre os psicofármacos mais utilizados.

Já em Campo Mourão, Paraná, nos anos de 2011 a 2013, em uma farmácia pública anexa a secretária de saúde, os medicamentos de controle especial mais dispensado, de acordo com as classes terapêuticas, foram os antidepressivos com total de 47,56\% (2011), 48,2\% (2012) e 46,7\% (2013) (PADILHA, 2014).

Esta predominância da classe de antidepressivos também foi evidenciada num estudo realizado no município de Ribeirão Preto/SP, em relação ao uso de antidepressivos e benzodiazepínicos, no qual a fluoxetina foi o antidepressivo mais prescrito e o Diazepam representou o ansiolítico mais prescrito na farmácia do SUS (NETTO, 2012).

Tal fenômeno não foi encontrado no atual trabalho, no qual predominou a classe dos antipsicóticos. Isso se deve a um viés encontrado na pesquisa, como comentando anteriormente, de que os CAPS atendem situações mais graves, sendo um serviço especializado em saúde mental, administrando os medicamentos em casos de emergências, é de responsabilidade das unidades de saúde (UBS) dispensar os medicamentos para tratamento contínuo e manejar casos que não sejam severos (PADILHA, 2014).

É descrito que os medicamentos psicotrópicos dispensados podem variar de acordo com a região, a especificidade do serviço e as necessidades da população envolvida (RIBEIRO, 2011).

Neste sentido, o perfil do consumo de medicamentos psicotrópicos em diferentes localidades do Brasil pode ser influenciado por diferentes fatores, como políticas de saúde, protocolos clínicos, aspectos sociais e culturais. Os resultados aqui apresentados referemse aos CAPS pesquisados em Curitiba/PR embora coincida com alguns dados obtidos em outros municípios brasileiros, porém discordam da premissa de que a fluoxetina é o medicamento psicotrópico mais utilizado no SUS (RIBEIRO, 2011; PADILHA, 2014).

\section{CONCLUSÃO}

O município tem um papel fundamental além de suprir a necessidade dos pacientes 
dispensando essas classes de medicamentos que compõe a REMUME (Relação Municipal de Medicamentos) baseada na RENAME. Porém, haja vista a complexidade de seus efeitos, torna-se necessário o correto diagnóstico, orientações adequadas ao paciente, e o acompanhamento farmacoterapêutico, pois esses medicamentos podem causar efeitos adversos e diferentes interações medicamentosas.

Desta forma, é importante ressaltar a presença do profissional farmacêutico também nas farmácias públicas, tanto para a organização dos fluxos dos medicamentos, estoques, registros legais, acesso, dispensação e do cuidado ao paciente. $O$ farmacêutico tem papel de referência para o apoio técnico-pedagógico às equipes para o uso racional dos medicamentos, evitando o uso inadequado de psicotrópicos no município, prestando um serviço de qualidade aos pacientes e contribuindo para a integralidade do cuidado.

Considerando tal fato, sugere-se a realização de pesquisa dos transtornos mentais mais prevalentes nos locais de estudo, bem como um levantamento sobre o acesso aos medicamentos prescritos, para que então seja possível cruzar tais dados com os até aqui obtidos, possibilitando avaliar a efetividade das farmacoterapias empregadas.

\section{AGRADECIMENTOS}

À Secretaria Municipal de Saúde de Curitiba (SMS), responsáveis pela indicação dos CAPS a serem estudados. Ao Ministério da Saúde pelo financiamento de bolsas no Programa de Educação pelo Trabalho (PET-Saúde, desenvolvido por UFPR/SMS/MS 2013-2015), e a Universidade Federal do Paraná, por nos dar toda estrutura física e cientifica necessária para realização do projeto.

\section{REFERÊNCIAS}

BAROZA, P.S.; SILVA, D.A. Medicamentos antidepressivos e antipsicóticos prescritos no centro de atenção psicossocial (CAPS) do município de Porciúncula- RJ. Acta Biom e- dica Brasiliensia, v. 3, n. 1, p. 85-97, 2012.

BRASIL. Portaria no 344, de 12 de maio de 1998. Aprova o Regulamento Técnico sobre substâncias e medicamentos sujeitos a controleespecial. Brasília: Diário Oficial da União, 31 de dezembro de 1998, Seção I. Disponível em: http://bvsms.saude.gov.br/bvs/saudelegis/ svs/1998/prt0344_12_05_1998_rep.html. Acesso em 05 de junho de 2016. 
BRASIL. Ministério da Saúde. Portaria no 3.088, de 23 de dezembro de 2011. Institui a Rede de Atenção Psicossocial para pessoas com sofrimento ou transtorno mental e com necessidades decorrentes do uso de crack, álcool e outras drogas, no âmbito do Sistema Único de Saúde (SUS). Diário Oficial de União, 2011. Disponível em:http://bvsms.saude.gov.br/bvs/saudelegis/gm/2011/prt3088_23_12_2011_rep.html. Acesso em 04 em junho de 2016.

BRASIL. Ministério da Saúde. Formulário Terapêutico Nacional. 2 $2^{\underline{a}}$ edição, Brasília: Ministério da Saúde, 2010.

BRASIL. Mistério da Saúde. Relação Nacional de Medicamentos Essenciais. 8aㅡ ed. Brasília: Ministério da Saúde, 2013.

CASTRO, C.G.S.O. Uma nova disciplina: a farmacoepidemiologia. In: . (Coord.). Estudos de utilização de medicamentos: noções básicas. Rio de Janeiro: Editora Fiocruz, 2000.

FILHO, P.C..T.; CHAGAS, A.G.; PINHEIRO, M.L.P.; LIMA, A.M.J.; DURÂO, M.A.S. Utilização de benzodiazepínicos por idosos para uma estratégia de saúde da família: implicações para enfermagem. Esc Anna Nery, n. 15, v.3, p.581-86, 2011.

FIRMINO, K.F.; ABREU, M.H.N.G.; PERINI, E.; MAGALHÃES, S.M.S. Utilização de benzodiazepínicos no serviço municipal de Saúde de Coronel Fabriciano, Minas Gerais. Cad Saúde Pública Coletiva, v. 17, v.1, p.157-66, 2012.

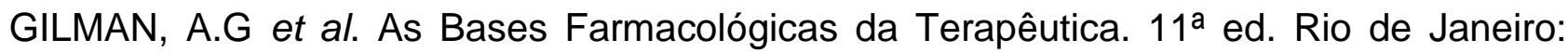
McGraw-Hill, 2006. 1436 p. GRAEFF, F.G.; GUIMARÃES, F.S.; Fundamentos da Psicofarmacologia. 2 $2^{\mathrm{a}}$ ed. São Paulo: Atheneu, 2012.

KANTAROSKI, L.P.; e.t al. Descrição de oferta e consumo dos psicofármacos em Centros de Atenção Psicossocial na Região Sul brasileira. Revista da Escola de Enfermagem da USP, n.45, v.6; p.1481-1487, 2011.

LOPES, L.M.B.; GRIGOLETO, A.R.L. Uso consciente dos psicotrópicos: responsabilidade dos profissionais de saúde. Braz J Health, n.2, v.1, p.1-14, 2011. 
NETTO, M.U.Q.; FREITAS, O.; PEREIRA, R.L.R. Antidepressivos e benzodiazepínicos: estudo sobre o uso racional entre os usuários do SUS de Ribeirão Preto - SP. Ver Ciênc Farm Básica, v.33, n.1, p.77-81, 2012.

OLIVEIRA, I. R. Antipsicóticos atípicos: farmacologia e uso clínico. Rev Bras Psiquiatria, n.22, v.l, p. 38-40, 2000.

ORLANDI, P.; NOTO, A. R. Uso indevido de benzodiazepínicos: um estudo com informantes-chave no município de São Paulo. Rev Latino-Am Enfermagem, n. 13, p. 896902, 2005.

PADILHA, P.D.M. Análise da dispensação de medicamentos psicotrópicos pela rede pública municipal de saúde de Campo Mourão/PR. Revista UNINGÁ, v.20, n.2, p.6-14, 2014.

RANG, H.P.; DALE, M.M.; RITTER, J.M.; MOORE, P.K. Farmacologia. $5^{\mathrm{a}}$ ed. Rio de Janeiro: Elsevier, 2004.

RIBEIRO, D. F.; LIMA, E.K.N.R. Possíveis interações farmacológicas entre psicotrópicos e a polioterapia realizada por pacientes adultos da cidade de Anápolis, Goiás [Monografia]. Goiás: Universidade Estadual de Goiás Unidade Universitária de Ciências exatas e tecnológicas; 2011.

RODRIGUES, M.A.P et al. Modificações nos padrões de consumo de psicofármacos em localidade do Sul do Brasil. Revista de Saúde Pública, n.40, v.1, p.107-114, 2016.

SESA. Secretaria de Estado da Saúde. Protocolo Clínico e Diretrizes Terapêuticas - Curitiba: SESA,2005. Disponível em: http://www.saude.pr.gov.br/modules/conteudo/conteúdo.php? conteudo=587. Acesso em: 28 de julho de 2015.

UNIVERSIDADE FEDERAL DO PARANÁ. Ministério da Saúde. Secretaria Municipal de Saúde de Curitiba. Programa de Educação pelo Trabalho para a Saúde/Redes de Atenção à Saúde - Universidade Federal do Paraná e Secretaria Municipal de Saúde de Curitiba 2013/2015 - Sub Projeto 4 - Rede de Atenção Psicossocial. Curitiba, 2013. 
WORLD HEALTH ORGANIZATION. Investing in Mental Health. Genebra: World Health Organization, 2003.

WORLD HEALTH ORGANIZATION. Relatório Mundial da Saúde - Saúde Mental: nova concepção, nova esperança. Lisboa: Climepsi Editores, 2001. 